\title{
Probabilistic finite element analysis of the uncemented hip replacement-effect of femur characteristics and implant design geometry
}

\author{
Carolina Dopico-González*, Andrew M. New, Martin Browne \\ Bioengineering Research Group, School of Engineering Sciences, University of Southampton, United Kingdom
}

\section{A R T I C L E I N F O}

Article history:

Accepted 27 September 2009

\section{Keywords:}

Total hip replacement

Finite element analysis

Probability analysis

\begin{abstract}
A B S T R A C T
In the present study, a probabilistic finite element tool was assessed using an uncemented total hip replacement model. Fully bonded and frictional interfaces were investigated for combinations of three proximal femurs and two implant designs, the Proxima short stem and the IPS hip stem prostheses. The Monte Carlo method was used with two performance indicators: the percentage of bone volume that exceeded specified strain limits and the maximum nodal micromotion. The six degrees of freedom of bone-implant relative position, magnitude of the hip contact force $(\mathrm{L})$, and spatial direction of $\mathrm{L}$ were the random variables. The distal portion of the proximal femurs was completely constrained and some of the main muscle forces acting in the hip were applied. The coefficients of the linear approximation between the random variables and the output were used as the sensitivity values. In all cases, boneimplant position related parameters were the most sensitive parameters. The results varied depending on the femur, the implant design and the interface conditions. Values of maximum nodal micromotion agreed with results from previous studies, confirming the robustness of the implemented computational tool. It was demonstrated that results from a single model study should not be generalised to the entire population of femurs and that bone variability is an important factor that should be investigated in such analyses.
\end{abstract}

(c) 2009 Elsevier Ltd. All rights reserved.

\section{Introduction}

There are many factors that need to be considered when analysing the performance of total hip replacement (THR) models. Both experimental and computational setups need to consider which of these factors are, in fact, important and which may be neglected. In order to reduce the expense of experimental simulations used to validate computational models, the latter should also act as a pre-experimental design tool to optimise its configuration, i.e. to find which parameters are more important to replicate and which values of these parameters are more representative or indicative of the behaviour of the system. This can be achieved through the implementation of probabilistic analyses together with finite element models. Some researchers have developed computational tools that enable analysis of multiple scenarios using a set of random variables simultaneously and efficiently (Browne and Langley, 1999; Nicolella et al., 2001; Bah and Browne, 2003; Perez et al., 2006; Easley et al., 2007; Mehrez, 2007). In probabilistic studies on THR models, it has been shown that there are many important random variables, such as bone geometry, implant design geometry, cement geometry, material properties, magnitude and direction of applied loads,

\footnotetext{
* Corresponding author.

E-mail address: cdg@soton.ac.uk (C. Dopico-González).
}

and bone-implant relative position that can affect performance (Browne and Langley, 1999; Nicolella et al., 2001; Bah and Browne, 2003; Perez et al., 2006; Easley et al., 2007; Mehrez, 2007; Dopico-Gonzalez et al., 2009a; Dopico-Gonzalez et al., 2009b; Halpern and Tanner, 1979; Heller et al., 2001; Herrlin et al., 1988; Laz et al., 2007a; Nicolella et al., 2006; Nishii et al., 2004; Reikeras et al., 1982; Speirs et al., 2007; Yang et al., 1984). This suggests that it is not appropriate to extrapolate the results obtained from simulation studies of one model to the whole population of femurs and implant designs. In a previous study (DopicoGonzalez et al., 2009a), a probabilistic investigation of a simplified finite element (FE) model of an uncemented THR was performed considering bone stiffness, implant stiffness, magnitude and spatial orientation of the applied load as the random variables. The maximum nodal von-Mises elastic strain was the performance indicator, and sensitivity results showed that bone stiffness was the most influential parameter, followed by load magnitude. In another study (Dopico-Gonzalez et al., 2009b), bone-implant version angle was included in the random variables and the percentage of bone volume that exceeded specified strain limits was also adopted as a performance indicator. This performance indicator gave more consistent results even for a low number of simulations and sensitivity results showed that bone-implant anteversion was one of the most influential parameters, together with load magnitude and bone stiffness. 
There were two objectives of the present study: (i) To further develop a computational tool (Dopico-Gonzalez et al., 2009a; Dopico-Gonzalez et al., 2009b) for the probabilistic analysis of the uncemented hip replacement by constructing a more realistic finite element (FE) model and by including 6 degrees of freedom of bone-implant relative position parameters in the set of random input variables and (ii) to assess the effect of femur characteristics and implant design geometry on the results. To this end, three femurs and two implant designs, the Proxima short stem (DePuy, Warsaw, USA) and the IPS stem (DePuy, Leeds, United Kingdom) were investigated using Monte Carlo-based simulations. Bone material properties were assigned to each element according to the CT scans of the femurs; forces due to some of the main muscle forces acting in the hip were applied and a convergence study was performed to decide on the optimum maximum element size. In previous studies, fully-bonded interfaces were assessed; in the present study frictional interfaces were also assessed to determine the effect of interface condition. The percentage of bone volume under a specified limit strain and the maximum nodal micromotion were adopted as performance indicators. Sensitivity analyses were performed based on the coefficients of a linear correlation between the input and the output parameters. Due to the complexity involved, the study does not attempt to recreate the in vivo mechanical environment exactly. However, a simplified but relevant replaced construct is utilized in order to enable a sufficient number of simulations to be run to perform a probabilistic analysis within the limits of available computational power and expense.

\section{Materials and methods}

\subsection{Finite element model}

The geometries of the three proximal femurs are shown in Fig. 1. These were generated by Radcliffe et al. (2007) using CT data and a commercially available software package (Mimics ${ }^{\mathbb{E}}$; Materialise NV, Leuven, Belgium). CT data were also used to determine material properties of the bones. All materials were assumed to have isotropic elastic properties.

The reference geometry was determined for the femur using MATLAB modules, as in a previous study (Dopico-Gonzalez et al., 2009a).

The femoral head cut was performed using a single plane. The position of this plane was different for each femur due to software limitations to perform the Boolean operations. The position and orientation parameters of the osteotomy planes are illustrated in Fig. 2, while the values of the parameters for the three femurs are presented in Table 1 .
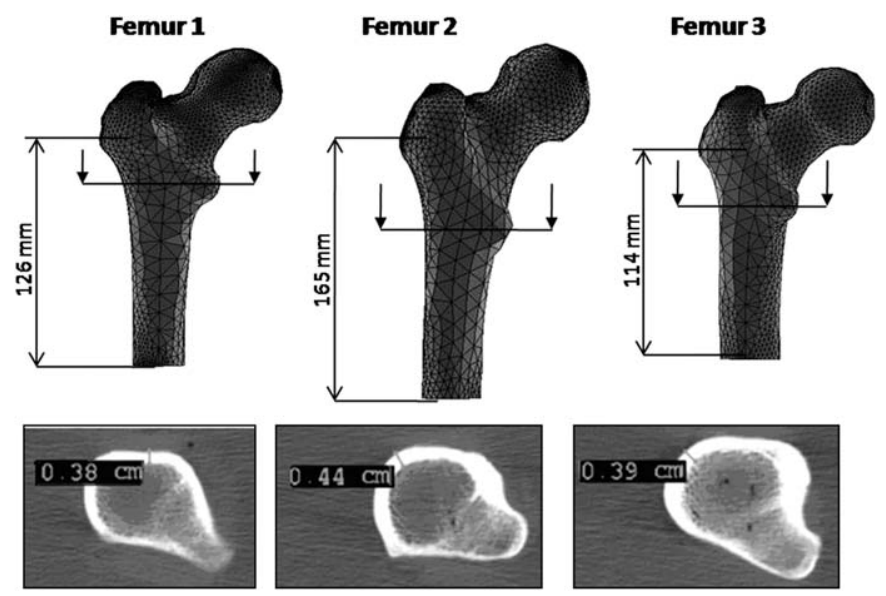

Fig. 1. Finite element models of the three proximal femurs (top) and cortical thickness in section at lesser trochanter level (bottom).

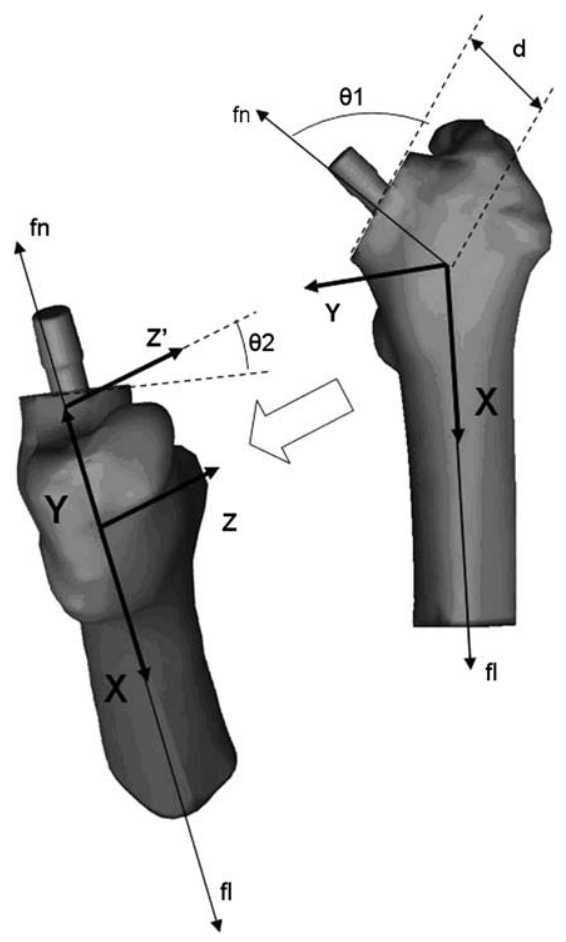

Fig. 2. Femoral axes (fl, fn) and parameters that define the osteotomy plane $(\theta 1, \theta 2)$.

Table 1

Values of the osteotomy position parameters for the three femurs.

\begin{tabular}{llrc}
\hline & $\boldsymbol{\theta 1}\left({ }^{\circ}\right)$ & $\boldsymbol{\theta 2}\left({ }^{\circ}\right)$ & $\boldsymbol{d}(\mathbf{m m})$ \\
\hline Femur 1 & 60 & 20 & 25 \\
Femur 2 & 60 & -20 & 25 \\
Femur 3 & 60 & 0 & 60 \\
\hline
\end{tabular}

The three bones were virtually implanted with the short-stem Proxima and the long-stem IPS (DePuy, Warsaw, IN, USA) (Fig. 3), both manufactured from titanium alloy, with a Young's modulus $(E)$ of $110 \mathrm{GPa}$ and a Poisson's ratio of 0.30 .

The implants were placed manually in reference positions such that the shaft axes of the femur and implant were almost parallel; the neck axes had an angle between $\pm 5^{\circ}$; and the distance between the approximated femoral head centre and the centre of the implant tapered neck section was of $\pm 2 \mathrm{~mm}$ in the local $X, Y$ and $Z$ directions.

For the six models, the geometries were built using a commercially available software package (Rhinoceros ${ }^{\mathbb{R}}$; McNeel, Seattle, USA), and the finite element meshes were generated using 4-noded tetrahedral elements in commercially available package (ANSYS-ICEMCFD ${ }^{\mathbb{R}}$; Ansys Inc, Canonsburg, PA, USA). A maximum element size of $6 \mathrm{~mm}$ was determined through a convergence study. The loads were applied and simulations run using a commercially available software package (Ansys $11.0^{\mathbb{R}}$; Ansys Inc.). The material properties of the bones were applied to the bone elements using a modified version of the freeware program BoneMat (Zannoni et al., 1998; Taddei et al., 2004) (Rizzoli Institute, Bologna, Italy). The relationship between the pixel intensity (Hounsfield units, HU) and the apparent density $\left(\rho, \mathrm{g} / \mathrm{cm}^{3}\right)$ was defined using calibration phantoms within the CT scans. These known values produced a linear relationship. The two points that describe this linear relationship were $(0,0.47)$ and $(1500,1.8)$. The apparent density of the voxels contained within each element was averaged and this value was used to determine $E$ (in $\mathrm{MPa}$ ) for each element, using the following expression (Keller, 1994):

\section{$E=10+2875 \rho^{3}$}

The muscle forces at the peak of the hip contact force measured during normal gait were applied. Some of the most important muscle forces in determining femoral bone strain during gait (gluteus minimus, gluteus medius, iliopsoas and vastus medialis) (Duda et al., 1998) were calculated using a commercially available 


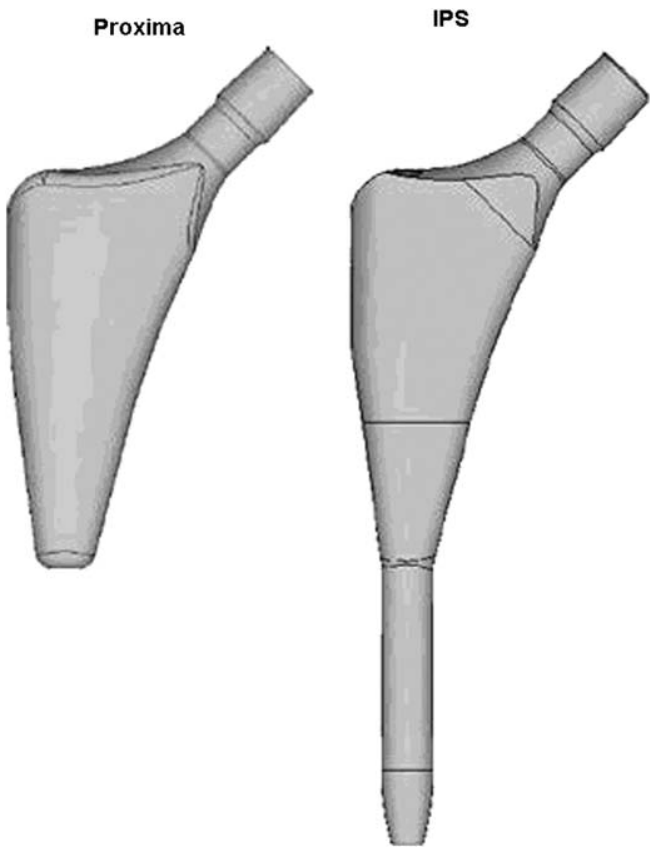

Fig. 3. Solid models of the Proxima and IPS implants.

software package (AnyBody ${ }^{\mathrm{B}}$; AnyBody Tech, Aalborg, Denmark) and a model validated by (Manders et al., 2008); these were applied to the FE models. The locations of the muscle attachment points were transferred from the AnyBody software, and the closest nodes to the location of these attachment points were selected for the application of the muscle forces. The values of these muscle forces are shown in Table 2 . The hip contact force (L) was applied at the implant node closest to the femoral head centre, the load magnitude and direction corresponded to the peak force measured for normal walking in an individual weighing $75 \mathrm{~kg}$ (Bergmann et al., 2001)

The bone-implant interface was defined with contact elements, in both fully bonded and frictional conditions. Four-noded surface-to-surface contact elements were defined to represent contact and sliding between the interface surfaces. The Augmented Lagrangian method was used as the contact algorithm (ANSYS). This method uses two factors: normal and tangent penalty stiffness (FKN and FKT, respectively). The FKN determines the amount of penetration between contact and target surfaces (ANSYS). A value of 0.5 was adopted to ensure convergence of the models with reasonable accuracy. A default value of FKT $=1$ was adopted. All the nodes in a distal portion of $10 \mathrm{~mm}$ thickness of the femoral model were constrained.

\subsection{Probabilistic model}

The random variables (RVs) considered in each model (Fig. 4) were: magnitude and spatial orientation of the hip contact force (L, ANGLY, ANGLZ); linear displacements of the prostheses with respect to the local femoral axes (OFFX, OFFY, OFFZ), and angular displacements of the prostheses with respect to the local femoral axes (ROTX, ROTY, ROTZ). Truncated uniform distributions were considered for the implant position parameters, with lower and upper bounds to ensure that the implant would not intersect the surface of the femur other than in the osteotomy section. The statistics of the RVs are shown in Table 3. The mean, upper and lower limit values corresponded to the peak values of $\mathrm{L}$ measured in normal walking for individuals weighing 75, 50 and $95 \mathrm{~kg}$, respectively (Bergmann et al., 2001). The load angle was varied to account for different loading conditions. 1000-trials Monte Carlo simulations were performed for each model and the performance indicators were the percentage of bone volume exceeding a vonMises elastic strain of $0.8 \%$ and the implant-bone maximum nodal micromotion in the frictional interface cases. This was calculated as the vectorial sum of the sliding and the gap of the nodes of the contact elements. Sensitivity parameters were calculated using a commercially available software (MATLAB R2008a ${ }^{\mathbb{R}}$; The MathWorks, Inc., Natick, MA, USA), based on the coefficients of linear correlations between the random variables and the performance indicator using the least squares method (Harter, 1983).

The probabilistic FE model was built using a set of software controlled by a Visual Basic algorithm, to allow free manipulation of the implant position in the bone. Rhinoceros (McNeel, Seattle, USA) was used to locate the implant at the desired position and to perform Boolean operations to generate the osteotomy and
Table 2

Values of the muscle forces applied to the models in Newtons (N) (Manders et al., 2008)

\begin{tabular}{|c|c|c|c|c|}
\hline & Femur 1 & Femur 2 & Femur 3 & \\
\hline Gluteus Minimus 1 & $\begin{array}{l}-93.41 \\
-11.0738 \\
-23.0719\end{array}$ & $\begin{array}{r}-86.1770 \\
-44.2855 \\
-2.4923\end{array}$ & $\begin{array}{r}-94.4750 \\
-9.4440 \\
-19.0338\end{array}$ & $\begin{array}{l}X \\
Y \\
Z\end{array}$ \\
\hline Gluteus Minimus 2 & $\begin{array}{r}-92.2757 \\
6.5574 \\
-37.0373\end{array}$ & $\begin{array}{l}-94.8074 \\
-27.3093 \\
-14.5135\end{array}$ & $\begin{array}{r}-93.3627 \\
7.3517 \\
-33.9954\end{array}$ & $\begin{array}{l}X \\
Y \\
Z\end{array}$ \\
\hline Gluteus Minimus 3 & $\begin{array}{r}-86.6447 \\
28.2256 \\
-56.9386\end{array}$ & $\begin{array}{r}-102.2642 \\
-4.8615 \\
-32.8921\end{array}$ & $\begin{array}{r}-87.8705 \\
27.7124 \\
-55.2688\end{array}$ & $\begin{array}{l}X \\
Y \\
Z\end{array}$ \\
\hline Gluteus Medius 1 & $\begin{array}{r}-221.1824 \\
-112.3301 \\
-33.6103\end{array}$ & $\begin{array}{r}-168.3625 \\
-185.2311 \\
5.8166\end{array}$ & $\begin{array}{r}-225.4163 \\
-107.1347 \\
-19.2420\end{array}$ & $\begin{array}{l}X \\
Y \\
Z\end{array}$ \\
\hline Gluteus Medius 2 & $\begin{array}{r}-177.4105 \\
-8.1806 \\
-37.7347\end{array}$ & $\begin{array}{r}-166.6315 \\
-72.2454 \\
2.4474\end{array}$ & $\begin{array}{r}-178.8517 \\
-4.7682 \\
-30.7728\end{array}$ & $\begin{array}{l}\mathrm{X} \\
\mathrm{Y} \\
\mathrm{Z}\end{array}$ \\
\hline Gluteus Medius 3 & $\begin{array}{r}-170.9275 \\
52.7976 \\
-82.1828\end{array}$ & $\begin{array}{r}-193.2293 \\
-12.6400 \\
-35.9681\end{array}$ & $\begin{array}{r}-172.3090 \\
53.4527 \\
-78.7677\end{array}$ & $\begin{array}{l}X \\
Y \\
Z\end{array}$ \\
\hline Iliopsoas & $\begin{array}{r}-481.8674 \\
-138.9206 \\
-24.6667\end{array}$ & $\begin{array}{r}-392.1126 \\
-305.7086 \\
70.3514\end{array}$ & $\begin{array}{r}-486.2308 \\
-125.1379 \\
0.6435\end{array}$ & $\begin{array}{l}X \\
Y \\
Z\end{array}$ \\
\hline Vastus Medialis & $\begin{array}{l}-0.2293 \\
-0.2536 \\
-0.3266\end{array}$ & $\begin{array}{l}-0.2867 \\
-0.2917 \\
-0.2833\end{array}$ & $\begin{array}{l}-0.2222 \\
-0.2739 \\
-0.3311\end{array}$ & $\begin{array}{l}X \\
Y \\
Z\end{array}$ \\
\hline
\end{tabular}

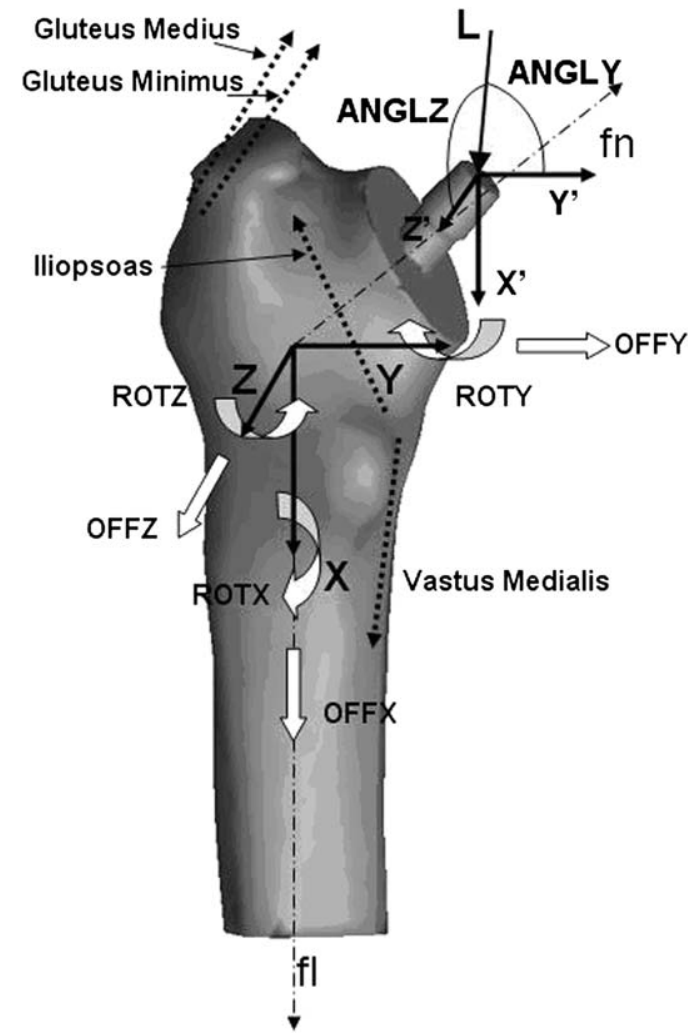

Fig. 4. FE model with the random variables (ANGLY, ANGLZ, L, OFFX, OFFY, OFFZ, ROTX, ROTY, ROTZ), the constant muscle loads and the local reference coordinate system.

the implant cavity. Ansys ICEM-CFD (Ansys Inc, Canonsburg, PA, USA) was used to repair the geometry and to mesh the construct. Ansys was used to assign material properties with Bonemat (Zannoni et al., 1998), apply all boundary conditions, define the bone-implant interface and solve the model. 


\section{Results}

The means and standard deviations of the output parameters (percentage of bone volume exceeding von-Mises elastic strain of

Table 3

Statistics of the random variables.

\begin{tabular}{lll}
\hline RV & Distribution & Mean (SD) [limit1, limit2] \\
\hline L $(\mathrm{N})$ & Truncated normal & $1775(260)[1200,2200]$ \\
ANGLY $\left(^{\circ}\right)$ & LogNormal & $90(30)$ \\
ANGLZ $\left(^{\circ}\right)$ & Truncated normal & $45(15)[0,90]$ \\
OFFX $(\mathrm{mm})$ & Uniform & {$[-3,3]$} \\
OFFY $(\mathrm{mm})$ & Uniform & {$[-2,2.5]$} \\
OFFZ $(\mathrm{mm})$ & Uniform & {$[-2.5,2.5]$} \\
ROTX $\left(^{\circ}\right)$ & Uniform & {$[-6,7]$} \\
ROTY $\left({ }^{\circ}\right)$ & Uniform & {$[-5,5]$} \\
ROTZ $\left(^{\circ}\right)$ & Uniform & {$[-5,5]$} \\
\hline
\end{tabular}

Proxima - Fully Bonded

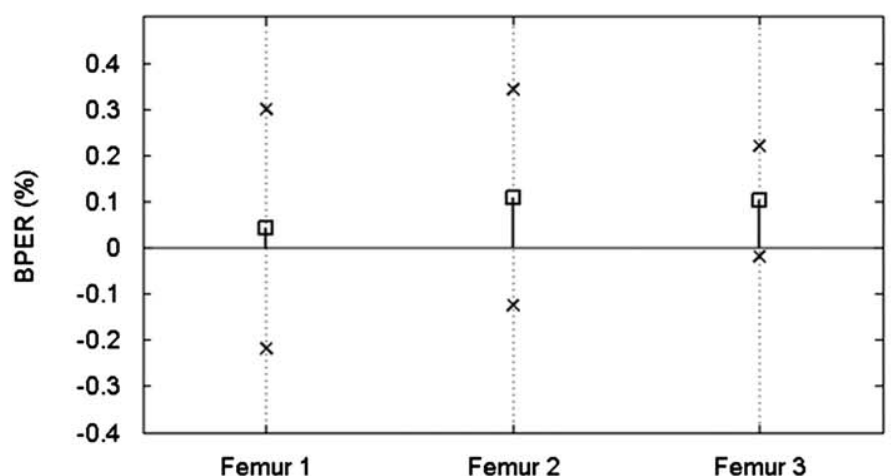

Proxima - Friction

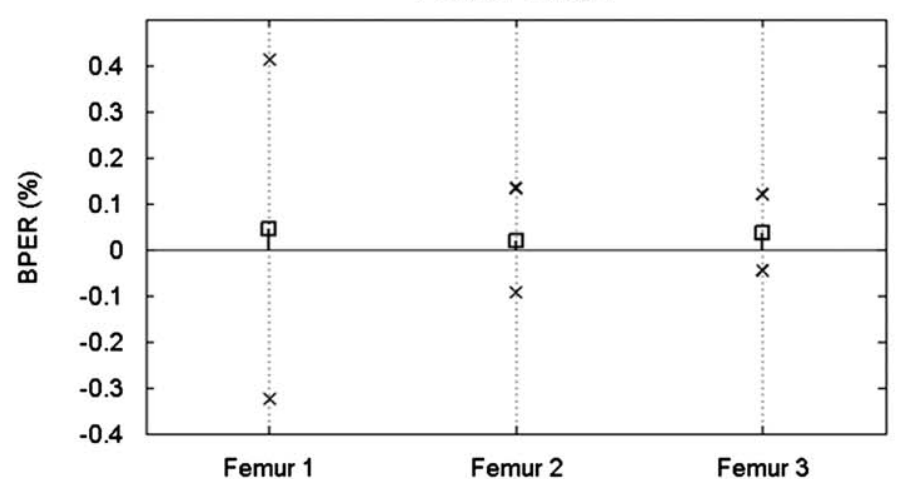

Proxima

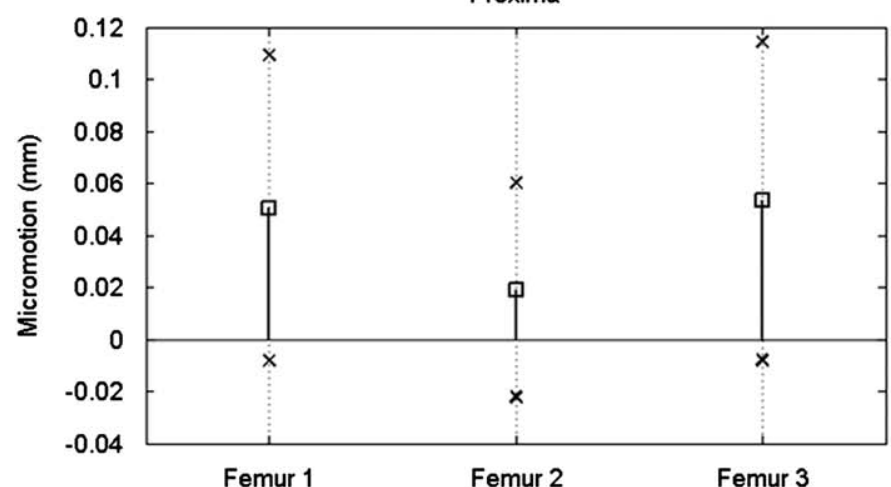

$0.8 \%$ (BPER) and maximum nodal micromotion) are presented in Fig. 5. The means and standard deviations of BPER were almost identical in most cases for fully bonded and frictional interface cases. Mean values of maximum nodal micromotion (Fig. 5 , bottom) were very similar for both implants, ranging from 20 to $50 \mu \mathrm{m}$ for the Proxima implant, and from 25 to $30 \mu \mathrm{m}$ for the IPS implant. Outlier values of maximum nodal micromotion were quite consistent for all the models, ranging from 277 to $299 \mu \mathrm{m}$.

The empirical cumulative distribution functions (CDFs) are presented in Fig. 6. For BPER, there was only a marginal difference between the curves for both interface cases, being identical for the Femur 3/IPS combination (centre, green lines). In general, the fully-bonded cases produced more convergence than the frictional interface cases, with the exception of Femur 2/Proxima combination. In both interface cases, most of the values of BPER ranged between 0 and $0.05 \%$. The CDFs of maximum nodal micromotion (bottom) were in excellent agreement for the three femurs. Femurs 1 and 3 with the Proxima implant produced
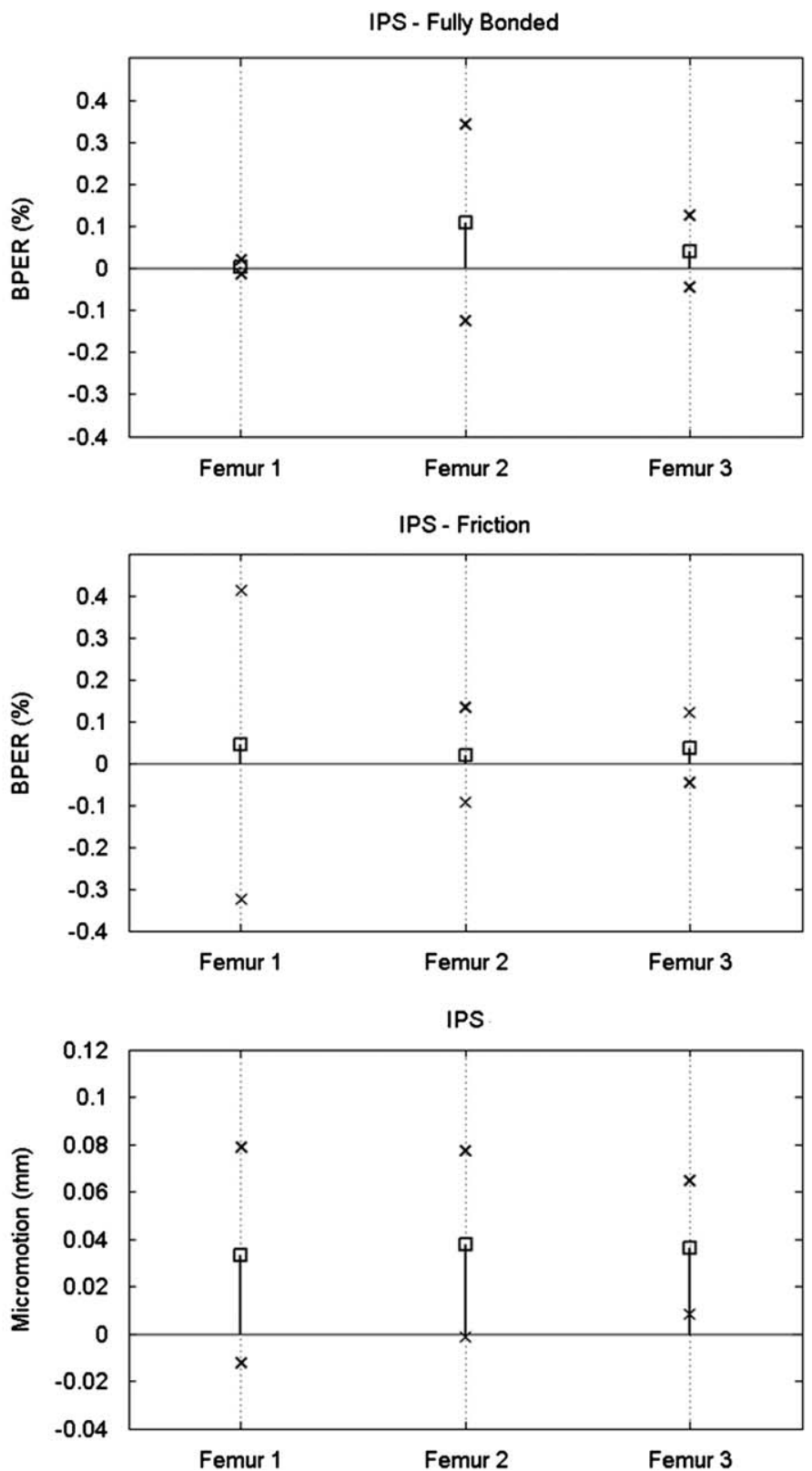

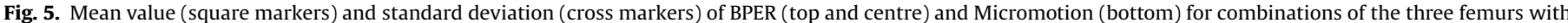
the Proxima (left) and the IPS (right) implants. Values of BPER are shown for fully-bonded (top) and frictional (centre) interface cases. 

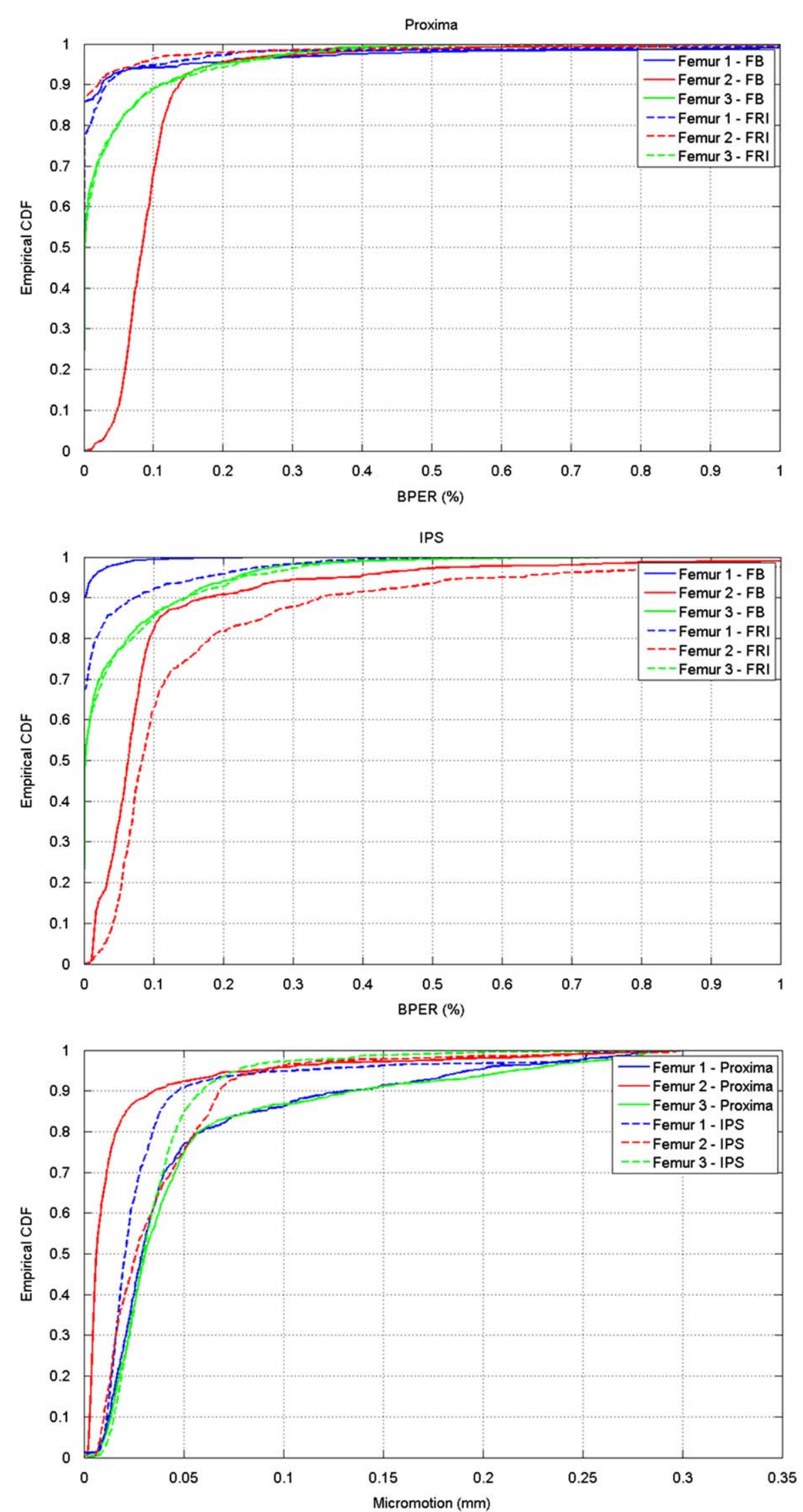

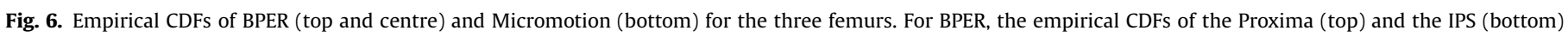
implants are shown, comparing the fully-bonded (top) and the frictional (centre) interface cases. 
identical CDFs. In addition the Proxima implant gave identical probabilities for values of micromotion $=50 \mu \mathrm{m}$ for the three femurs. For a probability of $50 \%$, the value of the maximum nodal micromotion was about $25 \mu \mathrm{m}$ for all the models.

The sensitivity results for BPER and maximum nodal micromotion are presented in Fig. 7. The trend of the sensitivities of BPER for each femur/implant combination was consistent for most of the models, except for Femur 1; when combined with the IPS prosthesis, the absolute values of the sensitivities of BPER were much lower than for the rest of the models, with a maximum for the sensitivity to OFFX (longitudinal direction). In addition, the sensitivities to load variability (ANGLY and ANGLZ) were higher than to implant positioning. Femur 1 combined with the Proxima prosthesis had a more consistent trend for both interface conditions, although the sensitivities to OFFY and to OFFZ were substantially decreased and increased, respectively. The remaining models presented similar sensitivities in both interface cases. Sensitivity to OFFY (medio-lateral direction) was one of the highest in all cases. Sensitivity to ROTZ (inclination)

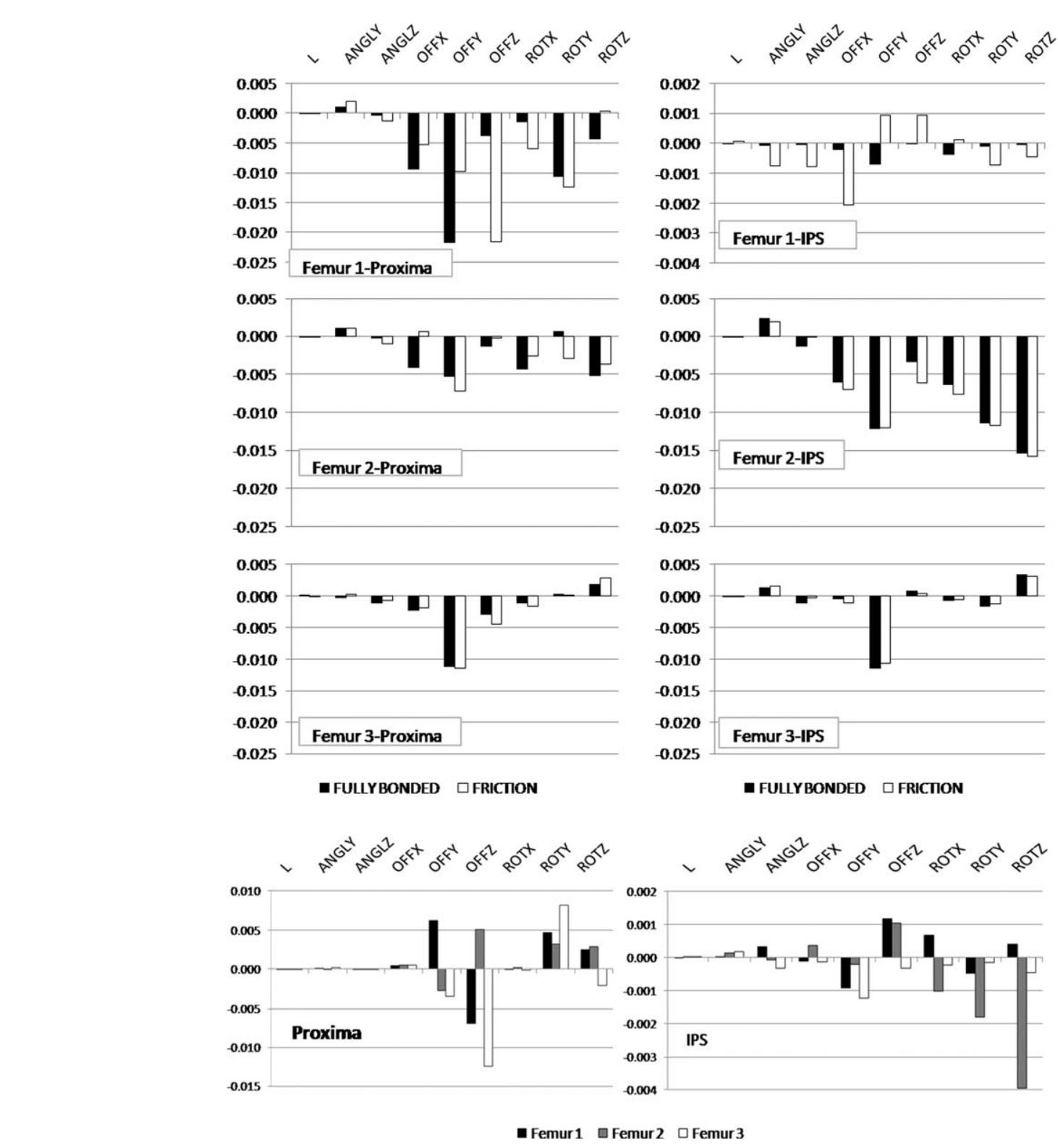

was also relatively high in all cases. Sensitivities for the maximum nodal micromotion output (bottom), showed that the Proxima prosthesis presented much higher sensitivities than the IPS prosthesis. In models with the Proxima implant, micromotion was most sensitive to OFFZ, followed by OFFY, ROTY and ROTZ, with femurs 1 and 3 the most sensitive in the majority of the cases. For the IPS prosthesis, Femur 2 had the highest sensitivities to ROTZ followed by ROTY, ROTX and OFFZ. Femur 1 had relatively high sensitivities, to OFFZ followed by OFFY, ROTX, ROTY and ROTZ. Femur 3 presented the minimum sensitivities, although OFFY was highly sensitive for both implants. In all cases, the maximum micromotion was most sensitive to implant positioning parameters than to load magnitude and geometric parameters.

\section{Discussion}

In order to benchmark the robustness of the implemented methodology, a series of parametric studies were performed. The

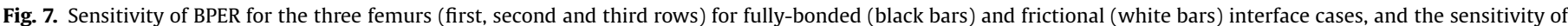
Micromotion for the three femurs (bottom) combined with the Proxima (left) and the IPS (right) implants. 
three femurs were selected from a database of 14 patients representing the range of minimum, median and maximum body mass indexes (BMI). The two implants were geometrically similar in the proximal region but differed in that only the IPS had a stem. The influence of stem length has been widely investigated due to the large number of implant geometries currently available; thus, the effect of this geometrical factor was assessed in this study. The effect of different bone-implant interface conditions was also assessed.

The values of the maximum nodal micromotion corresponded well with those of previous in vitro studies (Burke et al., 1991; Callaghan et al., 1992; Kassi et al., 2004). The average value of the maximum nodal micromotion for the IPS implant was under $30 \mu \mathrm{m}$, which is the maximum value that has been suggested for osseointegration to take place (Kassi et al., 2004). Furthermore, outlier values in previous studies (Burke et al., 1991) of the order of $280 \mu \mathrm{m}$ corresponded with those obtained in the present study (about $295 \mu \mathrm{m}$ for the Proxima implant and between 277 and $294 \mu \mathrm{m}$ for the IPS implant). These findings give confidence in the robustness of the methodology implemented in this study.

For a given femur, the sensitivities of BPER were the same for fully-bonded and frictional interface cases (Fig. 7, first, second and third rows). This suggests that the percentage of strained bone volume is similar within each femur/implant combination regardless of interface contact condition. These findings imply that it may be possible to run computational models of uncemented hip replacements using a fully-bonded interface for strain-based evaluations of the femur. This condition can be represented by merging the bone-implant interface, hence reducing significantly runtimes compared to models with interface contact definitions. From a clinical perspective, these findings suggest that the strain distribution in the femur may be similar at different stages of healing after a THR, regardless of implant positioning.

The geometry of the implant design clearly affected the sensitivities of maximum nodal micromotion. Changes in implant geometry resulted in changes in the geometry of the interface and consequently in the way the relative displacement between boneimplant, i.e., the micromotion, occurred. This suggests that the sensitivity of the micromotion is characteristic of the implant geometry. From a computational point of view, these findings imply that it is necessary to model different implant geometries to obtain holistic results. From a clinical point of view, this means that the healing after a THR, particularly immediately post operatively, will be affected differently by uncertainties depending on the implant geometry, i.e., it is more difficult to predict its behaviour.

Femur variability significantly affected the sensitivities of maximum nodal micromotion. Different bone geometry and stiffnesses influence the way the load is transmitted from the application point (implant node closest to the centre of the femoral head) to the bone-implant interface and hence the amount of micromotion. Computationally, this means that bone geometry and material variability should be considered in THR models to enable generalizations to the entire population of THRs. On the clinical side, this means that different patients will have a particular sensitivity to uncertainties during the healing process after surgery. In any case, sensitivity to implant positioning variability was shown to be high in all cases.

The average runtime of each model was $35 \mathrm{~h}$ for a full 1000 trial Monte Carlo simulation, using a $2 \mathrm{CPU}$ node at $3 \mathrm{GHz}$, with $72 \mathrm{~Gb}$ of RAM, running Red Hat Enterprise Linux $4^{\circledR}$. Several simplifications were necessary in this study in order to allow the analyses to be conducted in a reasonable run time: (i) the bones were assumed to be elastic and isotropic, as opposed to anisotropic (Ciarelli et al., 1991; Keaveny et al., 2001). Although it has been shown that non-linear material models more accurately predict the behaviour of bone (Keyak, 2001; Lotz et al., 1991b), some studies have shown that results from linear material models were also very similar (Cody et al., 1999; Lotz et al., 1991a). It has also been stated that non-linear models better represent the mechanical behaviour of the bone "past the onset of local material failure, whereas linear models are valid only up to the onset of failure" (Keyak, 2001). The output parameters used in the present study are representative of the risk of failure of the system, but they do not quantify failure as such represented by limit stress or strain to failure; rather they qualitatively represent the propensity for failure and at the same time reduce the computation time of the model; (ii) only the proximal section of the bones was considered and the bones were constrained in a region about $10 \mathrm{~mm}$ from the distal section. Many other studies have looked at similar models (Radcliffe et al., 2007; Keyak, 2001; Lotz et al., 1991a, 1991b; Decking et al., 2005; Keyak et al., 2001; Laz et al., 2007b; Lotz et al., 2005) recreating an in vitro configuration, where the distal femur is usually completely constrained (Burke et al., 1991; Decking et al., 2005; Britton and Prendergast, 2005); (iii) four-noded tetrahedral elements were selected instead of 10 -noded or hexahedral elements, which may have provided more accurate results. Although some authors have demonstrated that the hexahedral quadratic elements seemed to be more stable and less influenced to the degree of refinement (number of degrees of freedom) of the mesh (Ramos, 2006), it has also been found that tetrahedral linear element produced results close to theoretical ones (Ramos, 2006), and they are usually used since high quality meshes are much easier to generate automatically, which is one of the priorities of the present study; (iv) other sources of uncertainty could also have been considered, such as material properties, muscle forces variability, or osteotomy cut; however, due to available computational resources, only three random variables were considered, bone-implant relative position, load geometry and load magnitude; (v) for the same reasons only two performance indicators were considered, micromotion and a strain-based indicator, although other performance indicators could have been adopted, such as fracture load (Keyak, 2001; Keyak et al., 2001), stress distribution (Lotz et al., 2005; Laz et al., 2007b), fatigue limit (Easley et al., 2007) or strain distribution (Decking et al., 2005). The selection of the random variables in this study was thought to be appropriate given the challenge of representing implant position variability. In addition, further resources are needed if bone geometry and material uncertainties are to be considered, such as a substantial sample of bones, representative of the population. Implant material variability was found to be minimally sensitive in a previous study (Dopico-Gonzalez et al., 2009a) and hence it was neglected for the present study; some studies have suggested the use of strainbased failure criteria as fracture predictors (Wong et al., 2001; Kopperdahl, 1999; Schileo et al., 2007; Morgan and Keaveny, 2001; Bayraktar et al., 2004), similar to that adopted in the present work. It is assumed that the femur will break when a certain amount of bone suffers strains over a limit close to the yield strain, believed to be approximately $0.78 \% \pm 0.06$ (Kopperdahl, 1999). This performance indicator was also tested in a previous study and was found to be more robust (DopicoGonzalez et al., 2009b). The maximum nodal micromotion was also selected in order to assess an additional output relevant to the performance of the replaced construct. As computational resources and access to data improves, it would be useful to apply the aforementioned options (material anisotropy, alternative element types etc.) to refine and compare the FE models and the probabilistic outputs. The extent to which these limitations affect the conclusions of this study is subjective. On 
one hand, they have enabled the implementation of the technique in manageable computation times. On the other hand, they do highlight the evidence that both bone and implant variability affect the results. The refinement of the FE model should make the results more quantitative, as opposed to qualitative.

The present study has demonstrated a robust tool for the probabilistic analysis of the uncemented THR; this is affirmed by the good agreement with micromotion results from previous in vitro studies. This tool enables the multi-parameter assessment of THRs in much reduced times than with traditional parametric studies. With further improvements, this tool could be used as a pre-operative resource to decide on the most suitable implant for a specific patient. Both femur and implant geometry variability should be included in models of THRs in order to generalise the results to the entire population of THRs. If this is accomplished, with further refinement, this tool could be used to provide a holistic description of the response of different implant geometries during the design process.

\section{Conflict of interest statement}

This is part of a PhD thesis funded by DePuy, a Johnson \& Johnson Company, and the School of Engineering Sciences at the University of Southampton.

\section{Acknowledgement}

This research was supported by DePuy, a Johnson \& Johnson Company and the School of Engineering Sciences at the University of Southampton.

\section{References}

ANSYS, Release 10.1 Documentation

Bah, M.T. and Browne, M., 2003. Probabilistic analysis of a cemented hip implant, in International Congress on Computational Bioengineering 2003. Zaragoza, Spain.

Bayraktar, H.H., Morgan, E.F., Niebur, G.L., Morris, G.E., Wong, E.K., Keaveny, T.M., 2004. Comparison of the elastic and yield properties of human femoral trabecular and cortical bone tissue. Journal of Biomechanics 37, 27-35.

Bergmann, G., Deuretzbacher, G., Heller, M., Graichen, F., Rohlmann, A., Strauss, J., Duda, G.N., 2001. Hip contact forces and gait patterns from routine activities. Journal of Biomechanics 34, 859-871.

Britton, J., Prendergast, P.J., 2005. Preclinical testing of femoral hip components: an experimental investigation with four prostheses. Journal of Biomechanical Engineering 127 (5), 872-881.

Browne, M., Langley, R.S., Gregson, P.J., 1999. Reliability theory for load bearing biomedical implants. Biomaterials 20, 1285-1292.

Burke, D.W., O'Connor, D.O., Zalenski, E.B., Jasty, M., Harris, W.H., 1991. Micromotion of cemented and uncemented femoral components. Journal of Bone and Joint Surgery-British Volume 73, 33-37.

Callaghan, J.J., Fulghum, C.S., Glisson, R.R., Stranne, S.K., 1992. The effect of femoral stem geometry on interface motion in uncemented porous-coated total hip prostheses. Comparison of straight-stem and curved-stem designs. Journal of Bone and Joint Surgery American 74, 839-848.

Ciarelli, M.J., Goldstein, S.A., Kuhn, J.L., Cody, D.D., Brown, M.B., 1991. Evaluation of orthogonal mechanical properties and density of human trabecular bone from the major metaphyseal regions with materials testing and computed tomography. Journal of Orthopaedic Research 9, 674-682.

Cody, D.D., Gross, G.J., Hou, F.J., Spencer, H.J., Goldstein, S.A., Fyhrie, D.P., 1999. Femoral strength is better predicted by finite element models than QCT and DXA. Journal of Biomechanics 32, 1013-1020.

Decking, R., Puhl, W., Simon, U., Claes, L.E., 2005. Changes in strain distribution of loaded proximal femora caused by different types of cementless femoral stems. Clinical Biomechanics 21, 495-501.

Dopico-Gonzalez, C., New, A.M., Browne, M.A., 2009a. Computational tool for the probabilistic finite element analysis of an uncemented total hip replacement considering variability in bone-implant version angle. Computer Methods in Biomechanics and Biomedical Engineering 2009, 1-9.

Dopico-Gonzalez, C., New, A.M., Browne, M., 2009b. Probabilistic analysis of an uncemented total hip replacement. Medical Engineering and Physics 31, 470-476.
Duda, G.N., Heller, M., Albinger, J., Schulz, O., Schneider, E., Claes, L., 1998. Influence of muscle forces on femoral strain distribution. Journal of Biomechanics 31 841-846.

Easley, S., Pal, S., Tomaszewski, P., Petrella, A., Rullkoetter, P., Laz, P., 2007. Finite element-based probabilistic analysis tool for orthopaedic applications. Computer Methods and Programs in Biomedicine 85, 32-40.

Halpern, A.A., Tanner, J., Rinsky, L., 1979. Does persistent fetal femoral anteversion contribute to osteoarthritis?: a oreliminary report. Clinical Orthopedics Related Research Nov-Dec, 213-216.

Harter, H.L., (Ed.), S. Kotz and Johson, N.L. 1983. John Wiley \& Sons, Inc., New York, pp. 593-598.

Heller, M.O., Bergmann, G., Deuretzbacher, G., Claes, L., Haas, N.P., Duda, G.N., 2001 Influence of femoral anteversion on proximal femoral loading: measurement and simulation in four patients. Clinical Biomechanics 16 644-649.

Herrlin, K., Pettersson, H., Selvik, G., Lidgren, L., 1988. Femoral anteversion and restricted range of motion in total hip prostheses. Acta Radiologica 29, $551-553$.

Kassi, J.-P., Heller, M.O., Stoeckle, U., Perka, C., Duda, G.N., 2004. Stair climbing is more critical than walking in pre-clinical assessment of primary stability in cementless THA in vitro. Journal of Biomechanics 38, 1143-1154.

Keaveny, T.M., Morgan, E.F., Niebur, G.L., Yeh, O.C., 2001. Biomechanics of trabecular bone. Annual Review of Biomedical Engineering 3, 307-333.

Keller, T.S., 1994. Predicting the compressive mechanical behavior of bone. Journal of Biomechanics 27, 1159-1168.

Keyak, J.H., 2001. Improved prediction of proximal femoral fracture load using nonlinear finite element models. Medical Engineering and Physics 23, $165-173$.

Keyak, J.H., Rossi, S.A., Jones, K.A., Les, C.M., Skinner, H.B., 2001b. Prediction of fracture location in the proximal femur using finite element models. Medical Engineering and Physics 23, 657-664.

Kopperdahl, D.L., Keaveny, T.M., 1999. Yield strain behavior of trabecular bone Journal of Biomechanics 31, 601-608.

Laz, P.J., Stowe, J.Q., Baldwin, M.A., Petrella, A.J., Rullkoetter, P.J., 2007. Incorporating uncertainty in mechanical properties for finite element-based evaluation of bone mechanics. Journal of Biomechanics 40, 2831-2836.

Laz, P.J., Pal, S., Halloran, J.P., Baldwin, M.A., Langenderfer, J.E., Rullkoetter, P.J., 2007. Probabilistic Finite Element Analysis in Orthopaedic Biomechanics, in Bioengineering Modeling and Computer Simulation. CIMNE, Barcelona, Spain.

Lotz, J.C., Cheal, E.J., Haves, W.C., 1991a. Fracture prediction for the proximal femur using finite element models: part I-linear analysis. Journal of Biomechanical Engineering 113, 353-360.

Lotz, J.C., Cheal, E.J., Hayes, W.C., 1991b. Fracture prediction for the proximal femur using finite element models: part II-nonlinear analysis. Journal of Biomechanical Engineering 113, 361-365.

Lotz, J.C., Cheal, E.J., Hayes, C., 2005. Stress distributions within the proximal femur in gait and falls: implications for osteoporotic fracture. Osteoporosis International 5, 252-261.

Manders, C., New, A. and Rasmussen, J., 2008. Validation of musculoskeletal gait simulation for use in investigation of total hip replacement, in European Society of Biomechanics 2008. Luzerne (Switzerland).

Mehrez, L., 2007. The Application of Probabilistic Methods for the Assessment of Hip Implant Performance. Ph.D. Thesis 2007. Bioengineering Sciences Research Group. Southampton University.

Morgan, E.F., Keaveny, T.M., 2001. Dependence of yield strain of human trabecular bone on anatomic site. Journal of Biomechanics 34, 569-577.

Nicolella, D.P., Thacker, B.H., Katoozian, H., Davi, D.T., 2001. Probabilistic risk analysis of a cemented hip implant. American Society of Mechanical Engineers, Bioengineering Division 50, 427-428.

Nicolella, D.P., Thacker, B.H., Katoozian, H., Davy, D.T., 2006. The effect of threedimensional shape optimization on the probabilistic response of a cemented femoral hip prosthesis. Journal of Biomechanics 39, 1265-1278.

Nishii, T., Sugano, N., Miki, H., Koyama, T., Takao, M., Yoshikawa, H., 2004. Influence of component positions on dislocation. Computed tomographic evaluations in a consecutive series of total hip arthroplasty. Journal of Arthroplasty 19, $162-167$.

Perez, M.A., Grasa, J., Garcia-Aznar, J.M., Bea, J.A., Doblare, M., 2006. Probabilistic analysis of the influence of the bonding degree of the stem-cement interface in the performance of cemented hip prostheses. Journal of Biomechanics 39, 1859-1872.

Radcliffe, I., Prescott, P., Man, H.S., Taylor, M., 2007. Determination of suitable sample sizes for multi-patient based finite element studies. Medical Engineering \& Physics 29, 1065-1072.

Ramos, A., Simoes, J.A., 2006. Tetrahedral versus hexaedral finite elements in numerical modelling of the proximal femur. Medical Engineering \& Physics 28 916-924.

Reikeras, O., Bjerkreim, I., Kolbenstvedt, A., 1982. Anteversion of the acetabulum in patients with idiopathic increased anteversion of the femoral neck. Acta Orthopedica 53, 847-852.

Schileo, E., Taddei, F., Cristofolini, L. and Viceconti, M., 2007. Application of a Maximum Principal Strain Failure Criterion in Subject-Specific Finite Element Models of Bones, in III International Congress on Computational Bioengineering. 2007. Caracas, Venezuela. INABIO.

Speirs, A.D., Heller, M.O., Taylor, W.R., Duda, G.N., Perka, C., 2007. Influence of changes in stem positioning on femoral loading after THR using a shortstemmed hip implant. Journal of Clinical Biomechanics 22, 431-439. 
Taddei, F., Pancanti, A., Viceconti, M., 2004. An improved method for the automatic mapping of computed tomography numbers onto finite element models. Medical Engineering and Physics 26, 61-69.

Wong, A.S., Taylor, M., New, A.M. and Ritchie, A., 2001. Influence of an Interference Fit on the Strain Distribution in the Implanted Proximal Femur, in International Society of Biomechanics XVIIIth Congress 2001. Zurich, Switzerland.
Yang, R.J., Choi, K.K., Crowninshield, R.D., Brand, R.A., 1984. Design sensitivity analysis: a new method for implant design and comparison with parametric finite element analysis. Journal of Biomechanics 17, 849-854.

Zannoni, C., Mantovani, R., Viceconti, M., 1998. Material properties assignment to finite element models of bone structures: a new method. Medical Engineering and Physics 20, 735-740. 